\title{
COVID-19 Outbreak in Algeria: A Model to Predict Cumulative Cases
}

\author{
Mohamed Hamidouche ${ }^{1,2 *}$ (1)
}

${ }^{1}$ Pasteur Institute of Algeria, ALGERIA

${ }^{2}$ Public Health School of Pasteur, CNAM, Paris, FRANCE

*Corresponding Author: mohamidouche@hotmail.fr

Citation: Hamidouche M. COVID-19 Outbreak in Algeria: A Model to Predict Cumulative Cases. Journal of Contemporary Studies in Epidemiology and Public Health. 2020;1(1):ep20004. https://doi.org/10.30935/jconseph/8451

\begin{abstract}
Introduction: Since December 29, 2019 a pandemic of novel coronavirus-infected pneumonia named COVID-19 has started from Wuhan (China) and cases have been imported worldwide, in Algeria, the first case reported on February 25, 2020.

Methods: It is crucial to estimate the cases number growth in the early stages of the outbreak, to this end; we have implemented the Alg-COVID-19 Model, which allows predicting the incidence and the reproduction number R0 in the coming months in order to help decision makers. The initial equation (1) estimates the cumulative cases at (t) prediction time using two parameters: the reproduction number (R0) and the serial interval (SI).

Results: We found R0=2.55 (95\% CI 2.17-2.92) based on actual incidence of the first 26 days, using the serial interval $\mathrm{SI}=4,4$ and the prediction time $\mathrm{t}=26$. The herd immunity $\mathrm{HI}$ estimated is $\mathrm{HI}=61 \%$. Also, The predictions fits closely the actual incidence during the first 26 days of the epidemic in Algeria which allows us to use it. According to Alg-COVID-19 Model, the number of cases will exceed 5000 on the $42^{\text {nd }}$ day (April $7^{\text {th }}$ ) and it will double to 10000 on $46^{\text {th }}$ day of the epidemic (April $11^{\text {th }}$ ), thus, exponential phase will begin and increases continuously until reaching a herd immunity of $61 \%$ unless serious preventive measures are considered.

Discussion: This model is valid only when the majority of the population is vulnerable to COVID-19 infection, however, it can be updated to fit the new parameters values.

Keywords: coronavirus, COVID-19, modelisation, Algeria outbreak

Received: 17 Jun. $2020 \bullet$ Accepted: 29 Jul. 2020
\end{abstract}

\section{INTRODUCTION}

On December 29, 2019, Wuhan, the capital city of Hubei Province in Central China, has reported four cases of pneumonia with unknown etiology (unknown cause), the next day, the WHO China Country Office was informed [1] about this pneumonia cases that were found to have a link with Huanan seafood and animal market in Wuhan, the Centers for Disease Control and Prevention (CDC) and Chinese health authorities determined and announced later that a novel coronavirus denoted as Wuhan (CoV) had caused the pneumonia outbreak [2]. Since then, the outbreak has rapidly spread over a short span of time and has received considerable global attention.

On January 7, 2020 the etiological agent of the outbreak was identified as a novel coronavirus $(2019-\mathrm{nCoV})$ and its gene sequence was quickly submitted [3], renamed later SARS-CoV-2 and the coronavirus disease was renamed COVID-19 by WHO on February 12, 2020. It has since been identified as a zoonotic coronavirus, similar to SARS and MERS coronaviruses [4].
On January 30, 2020, WHO announced the listing of this Novel Coronavirus-infected Pneumonia (NCP) as a "public health emergency of international concern", a total of 254996 confirmed cases of infection with COVID-19, including 10444 deaths have been reported worldwide as on midday March 20, 2020 [5].

Sporadic cases have been imported to Europe, Africa and North and South America via returning travellers from China. In Algeria, the first case of COVID-19 was reported on February 25, 2020, when an Italian national tested positive in Ouargla region in the south of the country, a few days later, on March 1, 2020, two cases were reported in Blida region in the North of Algeria, following their contacts with two Algerian nationals who came from France for holidays, they were detected positive after their return to France, since then, a COVID-19 outbreak has started in this region (Blida) that form a cluster of more than 5.4 million inhabitants with the surrounding cities (Algiers, Boumerdes, Tipaza) [6,7], now, the epidemic is spreading to other parts of the country, until March 22, 2020, the Algerian authorities have declared 200 confirmed cases with a case fatality rate of $8.5 \%$ (17 deaths) [6]. 
Meanwhile, there is considerable uncertainty as to the extent of the epidemic and its parameters, the COVOD-19 reproduction number (R0) has been estimated in various studies, they found, 2,35 (95\% CI 1.15-4.77) [8], two studies used stochastic methods to estimate R0 have reported a range of 2.2 to 2.68 with an average of $2.44[9,10]$. Six studies that used mathematical methods to estimate R0 produced a range from 1.5 to 6.49 with an average of 4.2 [11-15], the three studies using statistical methods such as exponential growth estimated an R0 ranging from 2.2 to 3.58, with an average of 2.67 [16,17]. Also, the COVID-19 fatality rate vary by region from $0.39 \%$ in Norway to $8,3 \%$ in Italy, in China the case fatality rate is $4 \%[18,19]$, however, the highest case fatality rate was reported in Algeria, reaching 8.5\% until March 22, 2020 [6].

In the early stages of a new infectious disease outbreak, as COVID19 in Algeria, it is crucial to estimate the transmission dynamics and inform predictions about potential future epidemic growth [20], it can provide insights into the epidemiological situation which helps decision makers to adapt the health system capacities. Thus, a prediction model can help to do that and identify whether outbreak control measures are having a measurable effect or not [21,22] and guide the design of alternative interventions [23]. In addition, a prediction model can be updated to help estimate risk to other countries [24].

To this end, we have implemented the Alg-COVID-19 Model which allows to predict the cumulative cases in the coming weeks, and to calculate the actual basic reproduction number (R0). Consequently, this model can show hospitals what to expect in terms of COVID-19 patients, amongst, the percentage of those who need to be in an intensive care unit (ICU) or on a ventilator, and also, the future number of deaths based on a given data.

\section{METHODS}

\section{Epidemiological data}

We retrieved information on cases number with confirmed COVID-19 infection based on official reports from governmental institutes in Algeria [6].

\section{The Model Equation}

This model Alg-COVID-19 only considers susceptible and infected subjects (SI model) without taking into account the recovered people, so, to predict the cumulative cases of COVID-19 Algerian epidemic in the coming weeks we used the equation defined as:

$$
I(t)=p \times R 0^{t / S I}
$$

I $(t)$ : The incidence (cumulative cases number) at $t$ time

R0: The basic reproduction number

SI: The serial interval

$\mathrm{t}$ : The prediction time

p: The baseline prevalence

Estimation of Basic Reproduction Number (R0) and the Herd Immunity

The basic reproduction number is defined as the (average) number of new infections generated by one infected individual during the entire infectious period in a fully susceptible population. It can be also understood as the average number of infections caused by a typical individual during the early stage of an outbreak when nearly al individuals in the population are susceptible to infection. The basic reproduction number reflects the ability of an infection spreading under no control, it has three components [25]:

$$
R 0=C B * N * D
$$

CB: effective contact rate (C: contact frequency, B: contact efficacy)

$\mathrm{N}$ : susceptible population size

D: infectious phase duration

The approach implemented to estimate the basic reproduction numbers (R0) in this model is to calculate the average R0 using the actual chronological cumulative cases during the first 26 days of the COVID-19 Algeria epidemic between February 25 and March 22, 2020, so that, we used the equation (3) derived from the equation (1). The R0 will be used also to calculate the herd immunity (HI) needed to stop the epidemic spontaneously based on the equation 4 .

$$
\begin{gathered}
R 0 \text { Average }=\left[\sum \operatorname{Exp}^{\left(\frac{\log [I(t) / p]}{t / S I}\right)}\right] \div 26 \\
H I=\left(1-\frac{1}{R 0}\right) * 100 \%
\end{gathered}
$$

\section{Estimation of the Serial Interval (SI)}

The serial interval (SI) is the time between symptom onset of a primary and secondary case, a previous studies reported that (SI = $4.4 \pm 3.17$ ) days [13], so we used this value in our model to predict the incidence.

\section{Statistical Analysis and Software}

This study was conducted using Excel 2013 and STATA/IC 15 software.

\section{RESULTS}

\section{The Initial Parameters Used in Alg-COVID-19 Model}

Without considering the prevention measures and other factors, this paper focused on the predicted COVID-19 incidence at any time of the epidemic using Alg-COVID-19 Model (equation 1), based on three parameter combinations that create plausible epidemic curves, namely, the average basic reproduction number calculated with the actual cases number at the first 26 days R0=2.55 (95\% CI 2.17-2.92) (equation 3), the serial interval $(\mathrm{SI}=4.4)$ and the prediction time $(\mathrm{t})$ we are looking for. The herd immunity (HI) estimated using the (equation 4) is $\mathrm{HI}=61 \%$.

\section{Model Fitting}

The COVID-19 incidence (cases number) predicted with the AlgCOVID-19 Model fits closely the actual incidence during the first 26 days of the epidemic in Algeria (Figure 1A), this allows us to use this model to predict the COVID-19 incidence in the next months of the epidemic (Figure 1B). 


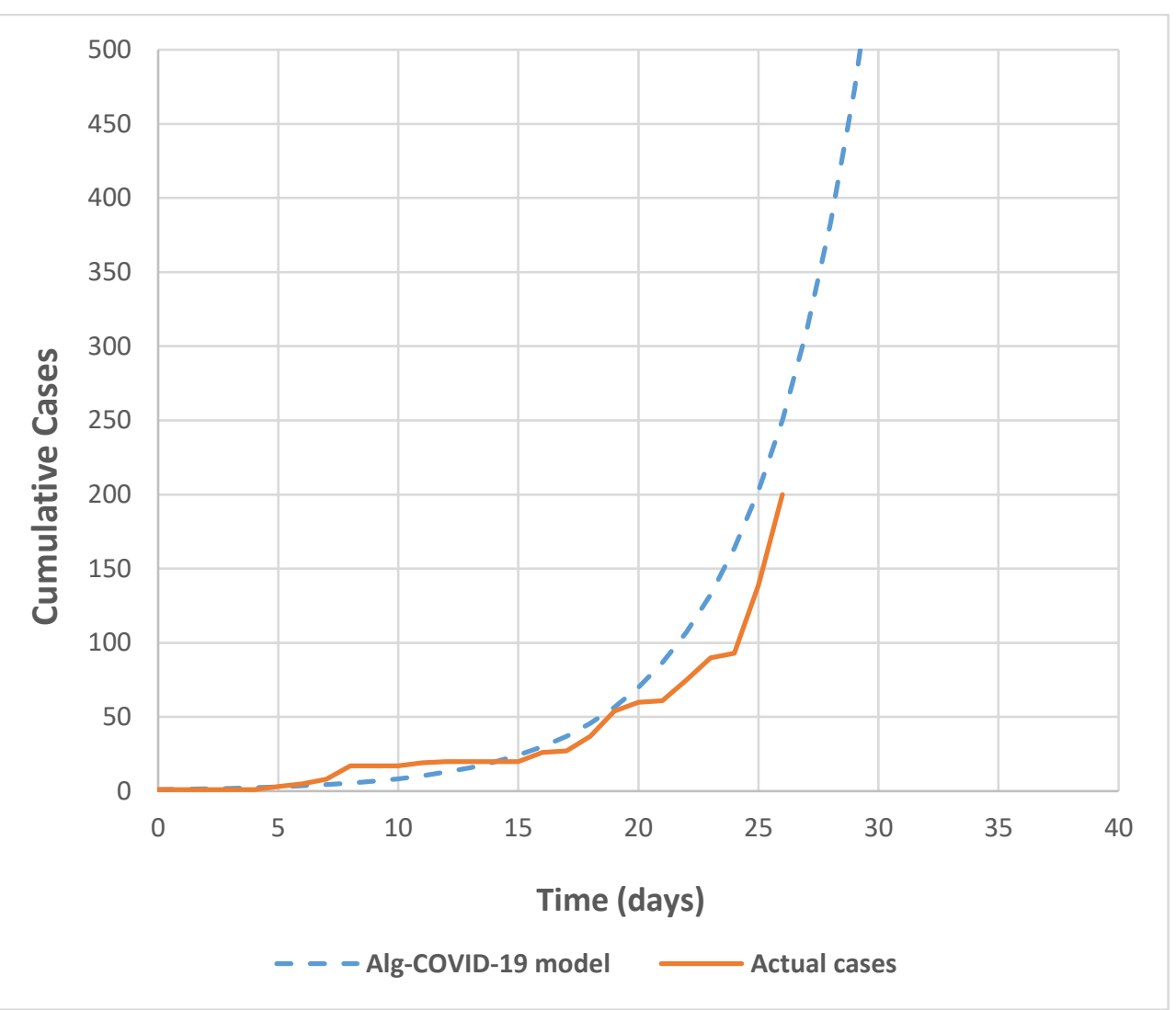

A

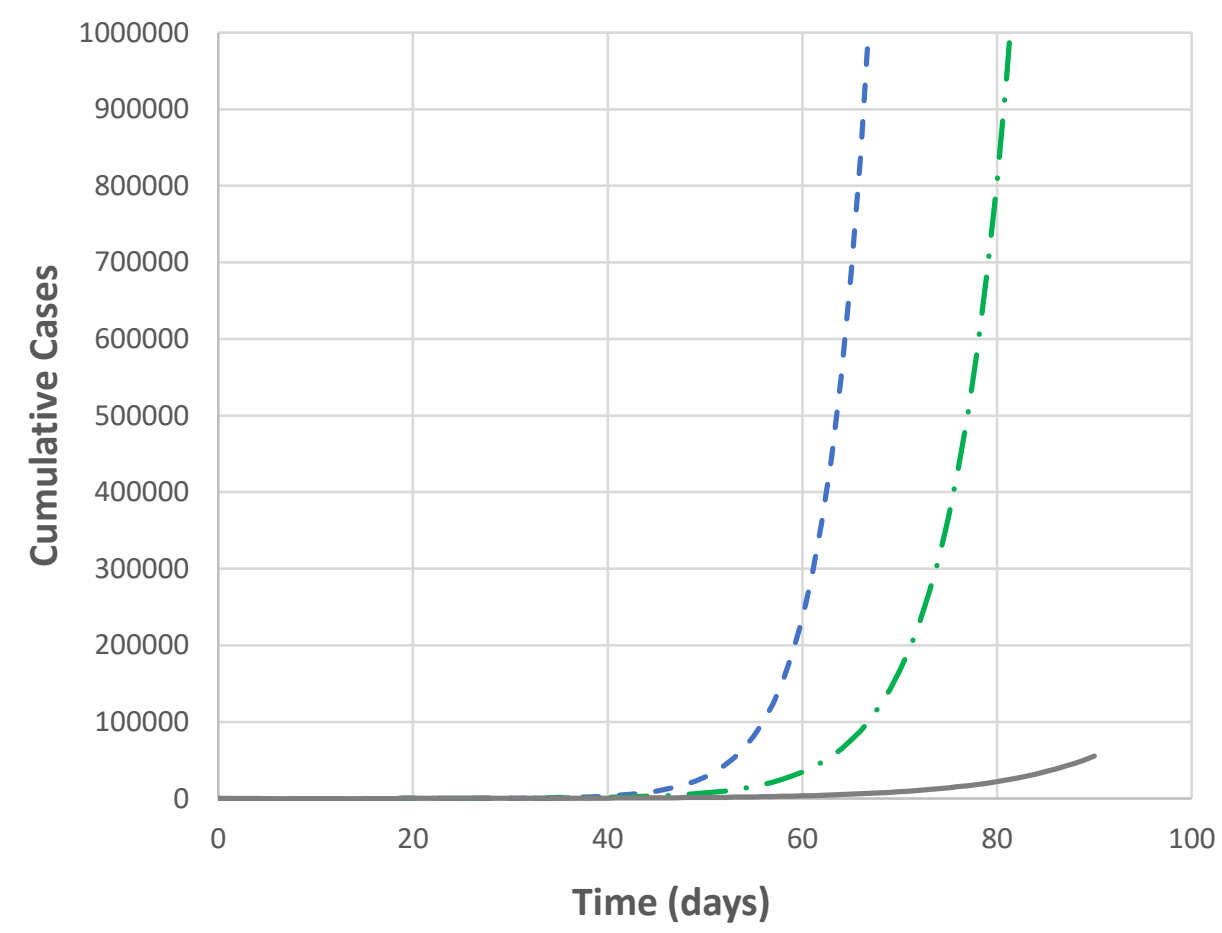

- - Alg-COVID-19 Model (R0=2.55) — - Alg-COVID-19 Model (R0=2) Alg-COVID-19 Model (R0=1.5)

Figure 1. A) Alg-COVID-19 Model match to actual data (cumulative cases) of epidemic's first 26 days. B) Alg-COVID-19 Model before mitigation $(\mathrm{R} 0=2.55)$, and after mitigation $(\mathrm{R} 0<2.55)$ 
Table 1. Alg-COVID-19 Model results for next weeks without considering the preventive measures $(\mathrm{R} 0=2.55)$ and with preventive measures $(\mathrm{R} 0=2$ and $\mathrm{R} 0=1.5)$

\begin{tabular}{|c|c|c|c|c|}
\hline Date & Days & Cases $(\mathrm{R} 0=2,55)$ & Cases $(\mathrm{R} 0=2)$ & Cases $(\mathrm{R} 0=1,5)$ \\
\hline $21 / 03 / 2020$ & 25 & 139 & 139 & 139 \\
\hline $22 / 03 / 2020$ & 26 & 172 & 163 & 152 \\
\hline $23 / 03 / 2020$ & 27 & 213 & 190 & 167 \\
\hline $24 / 03 / 2020$ & 28 & 263 & 223 & 183 \\
\hline $25 / 03 / 2020$ & 29 & 325 & 261 & 201 \\
\hline $26 / 03 / 2020$ & 30 & 402 & 306 & 220 \\
\hline $27 / 03 / 2020$ & 31 & 497 & 358 & 242 \\
\hline $28 / 03 / 2020$ & 32 & 615 & 419 & 265 \\
\hline $29 / 03 / 2020$ & 33 & 760 & 490 & 290 \\
\hline $30 / 03 / 2020$ & 34 & 940 & 574 & 319 \\
\hline $31 / 03 / 2020$ & 35 & 1163 & 672 & 349 \\
\hline $01 / 04 / 2020$ & 36 & 1438 & 786 & 383 \\
\hline $02 / 04 / 2020$ & 37 & 1779 & 920 & 420 \\
\hline $03 / 04 / 2020$ & 38 & 2199 & 1077 & 461 \\
\hline $04 / 04 / 2020$ & 39 & 2720 & 1261 & 505 \\
\hline $05 / 04 / 2020$ & 40 & 3364 & 1477 & 554 \\
\hline $06 / 04 / 2020$ & 41 & 4160 & 1728 & 607 \\
\hline $07 / 04 / 2020$ & 42 & 5145 & 2023 & 666 \\
\hline $08 / 04 / 2020$ & 43 & 6362 & 2369 & 730 \\
\hline $09 / 04 / 2020$ & 44 & 7868 & 2773 & 800 \\
\hline $10 / 04 / 2020$ & 45 & 9730 & 3246 & 878 \\
\hline $11 / 04 / 2020$ & 46 & 12033 & 3799 & 963 \\
\hline $12 / 04 / 2020$ & 47 & 14882 & 4448 & 1055 \\
\hline $13 / 04 / 2020$ & 48 & 18404 & 5207 & 1157 \\
\hline $14 / 04 / 2020$ & 49 & 22759 & 6095 & 1269 \\
\hline $15 / 04 / 2020$ & 50 & 28146 & 7135 & 1393 \\
\hline $16 / 04 / 2020$ & 51 & 34807 & 8353 & 1526 \\
\hline $17 / 04 / 2020$ & 52 & 43045 & 9778 & 1673 \\
\hline $18 / 04 / 2020$ & 53 & 53233 & 11446 & 1835 \\
\hline $19 / 04 / 2020$ & 54 & 65832 & 13399 & 2012 \\
\hline $20 / 04 / 2020$ & 55 & 81413 & 15685 & 2206 \\
\hline $21 / 04 / 2020$ & 56 & 100681 & 18361 & 2419 \\
\hline $22 / 04 / 2020$ & 57 & 124510 & 21494 & 2653 \\
\hline $23 / 04 / 2020$ & 58 & 153979 & 25162 & 2909 \\
\hline $24 / 04 / 2020$ & 59 & 190423 & 29455 & 3189 \\
\hline $25 / 04 / 2020$ & 60 & 235491 & 34480 & 3497 \\
\hline $26 / 04 / 2020$ & 61 & 291227 & 40363 & 3835 \\
\hline $27 / 04 / 2020$ & 62 & 360153 & 47250 & 4205 \\
\hline $28 / 04 / 2020$ & 63 & 445392 & 55312 & 4611 \\
\hline $29 / 04 / 2020$ & 64 & 550806 & 64749 & 5056 \\
\hline $30 / 04 / 2020$ & 65 & 681169 & 75797 & 5544 \\
\hline
\end{tabular}

\section{Alg-COVID-19 Model Results}

The estimations presented by this model are based on the first 26 days data and cover the next months of COVID-19 epidemic in Algeria, according to that, the number of cases will exceed 1000 cases on the $35^{\text {th }}$ day of the epidemic (March 31, 2020), 5000 on the $42^{\text {nd }}$ day (April $7^{\text {th }}$ ) and it will double to 10000 on $46^{\text {th }}$ day of the epidemic (April $\left.11^{\text {th }}\right)$, thus, exponential phase will increases continuously until reaching à herd immunity of $61 \%$ or a serious preventive measures are considered (Table 1; Fig ure 1B).
DISCUSSION

Regarding the used parameters (R0 and SI) by Alg-COVID-19 Model for the predictions, the estimated $\mathrm{R} 0=2.55$ (95\% CI 2.17-2.92) until March 19, 2020, is very close to R0 that was found on the Chinese epidemic data $\mathrm{R} 0=2,35$ (95\% CI, 1.15-4.77) until February $4^{\text {th }}, 2020$ [8], also, it is in the range of basic reproduction numbers of other studies mentioned below [11-15], on the other hand, the parameter that we cannot update and can affect the predictions is the serial interval (SI), we found two studies each one has different value that range from 
4.4 \pm 3.17 [13] to 7.5 days (95\% CI, 5.3-19) [26], we used (SI=4.4) because of its standard deviation is not very large.

This model only considers susceptible and infected subjects (SI model) without taking into account the recovered people, so, it is valid only when the majority of the population is vulnerable to COVID-19 infection, without taking into consideration the herd immunity acquired by recovered people ongoing epidemic and the ability of getting infected a second time.

In case of R0 decreases over the next weeks of the epidemic, AlgCOVID-19 Model can be revalidated by recalculating a new effective reproductive number R1 (equation 3). However, this will happen only if the herd immunity rises spontaneously during the epidemic or if serious preventive measures acting directly on one of the components of R0 will be taken (equation 2).

In the case of the Algerian COVID-19 epidemic, this model can be valid for the first few months if the herd immunity remains low and susceptible population size c change, thus, it is applicable whenever a new regional outbreak begins with a naive population. On the other hand, if the state implements a good preventive policy according to the WHO guidelines [5] and set up measures to reduce the effective contact rate, this will obviously reduce R0, so that, (R1) the effective reproduction number with control and preventive measures can be calculated to estimate the implemented preventive actions effectiveness and performance. The Alg-COVID-19 Model can be updated with R1 values adapted to the new situation and its forecasts would correspond as closely as possible to the real epidemic growth (Figure 1B). Furthermore, it is obvious that the measures must be strong enough to reduce the basic reproduction number below $1(\mathrm{R} 0<1)$ to control the epidemic, otherwise, it will spread over time, which can also be a good strategy for maintaining the healthcare demand at a manageable pace.

According to Alg-COVID-19 Model results based on Algerian data without taking into account the preventive measures $(\mathrm{R} 0=2.55)$, the exponential phase will begin after the $35^{\text {th }}$ day of the epidemic (1000 cases) by March 31, 2020, consequently, the response to the epidemic will change from trying to curb the spread of the COVID-19 on the territory to a strategy that lead to mitigate the effects of the epidemic waves, so that, after R0 and SI update (Figure 1B), the model can be used to predict the number of; hospitalization, ICU or under ventilator patients, and also, to predict the number of deaths that remains the highest in the wold (Algeria: $8.5 \%$ until March $22^{\text {nd }}$ ) since the start of the pandemic, without any concrete explanation. Predictions with preventive measures at $\mathrm{R} 0=2$ and $\mathrm{R} 0=1.5$ are also presented in Table 1.

\section{REFERENCES}

1. WHO (2020). Available at: https://www.who.int/csr/don/05january-2020-pneumonia-of-unkown-cause-china/en/

2. CDC. 2020. Available at: https://www.cdc.gov/coronavirus/ novel-coronavirus-2019.html

3. GenBank. 2019. Available at: http://virological.org/t/novel-2019coronavirus-genome/319
4. Liu Y, Gayle AA, Wilder-Smith A, Rocklöv J. The reproductive number of COVID-19 is higher compared to SARS coronavirus. Journal of Travel Medicine, 2020;27(2):taaa021. (doi: 10.1093/jtm/taaa021).

5. WHO (2020). Available at: https://www.who.int/emergencies/ diseases/novel-coronavirus-2019/technical-guidance

6. Algerian Ministry of Health. 2020. Available at: http://covid19.sante.gov.dz/carte/

7. ONSA. Office national des statistiques d'Algérie. 2020. Available at: http://www.ons.dz/

8. Kucharski AJ, Russell TW, Diamond C, Liu Y, et al. Early dynamics of transmission and control of COVID-19: a mathematical modelling study. Lancet Infect Dis Published Online March 11, 2020;2020(20)30144-4. (doi: 10.1016/S1473-3099(20)30144-4).

9. Wu JT, Leung K, Leung GM. Nowcasting and forecasting the potential domestic and international spread of the 2019-nCoV outbreak originating in Wuhan, China: a modelling study. The Lancet, 2020;395(10225):689-97. (doi: 10.1016/S01406736(20)30260-9).

10. Riou J, Althaus CL. Pattern of early human-to-human transmission of Wuhan 2019-nCoV. bioRxiv, 2020. (doi: 10.1101/2020.01.23.917351).

11. Shen M, Peng Z, Xiao Y, Zhang L. Modelling the epidemic trend of the 2019 novel coronavirus outbreak in China. bioRxiv, 2020. (doi: 10.1101/2020.01.23.916726).

12. Read JM, Bridgen JRE, Cummings DAT, Ho A, Jewell CP. Novel coronavirus 2019-nCoV: early estimation of epidemiological parameters and epidemic predictions. medRxiv, 2020. (doi: 10.1101/2020.01.23.20018549).

13. You C, Deng Y, Hu W, Sun J, Lin Q, Zhou F, et al. Estimation of the Time-Varying Reproduction Number of COVID-19 Outbreak in China. medRxiv, 2020. (doi: 10.1101/2020.02.08.20021253).

14. Imai N, Cori A, Dorigatti I, Baguelin M, et al. Report 3: Transmissibility of 2019-nCoV. COVID-19 Response Team, Imperial College London, 2020. (doi: 10.25561/77148).

15. Tang B, Wang X, Li Q, Bragazzi NL, et al. Estimation of the Transmission Risk of 2019-nCov and Its Implication for Public Health Interventions. J Clin Med., 2020 Feb;9(2):462. (doi: 10.3390/jcm9020462). 
16. Zhao S, Lin Q, Ran J, Musa SS, et al. Preliminary estimation of the basic reproduction number of novel coronavirus (2019-nCoV) in China, from 2019 to 2020: A datadriven analysis in the early phase of the outbreak. Int J Infect Dis. 2020 Mar;92:214-7. (doi: 10.1016/j.ijid.2020.01.050).

17. Liu T, Hu J, Kang M, Lin L, et al. Transmission dynamics of 2019 novel coronavirus (2019-nCoV). bioRxiv, 2020. (doi: 10.1101/2020.01.25.919787)

18. Wilson N, Kvalsvig A, Telfar L, Baker MG. Case-Fatality Risk Estimates for COVID-19 Calculated by Using a Lag Time for Fatality. Emerg Infect Dis., 2020;26(6):1339-441. (doi: 10.3201/eid2606.200320).

19. Johns Hopkins. 2003 2020. Available at: https://coronavirus.jhu.edu/

20. Viboud C, Sun K, Gaffey R, Ajelli M, et al. The RAPIDD Ebola forecasting challenge: synthesis and lessons learnt. Epidemics, 2018;22:13-21. (doi: 10.1016/j.epidem.2017.08.002).

21. Funk S, Ciglenecki I, Tiffany A, Gignoux E, et al. The impact of control strategies and behavioural changes on the elimination of Ebola from Lofa County, Liberia. Philos Trans R Soc Lond B Biol Sci, 2017;372(1721): 20160302. (doi: 10.1098/rstb.2016.0302).
22. Riley S, Fraser C, Donnelly CA, et al. Transmission dynamics of the etiological agent of SARS in Hong Kong: impact of public health interventions. Science, 2003;300:1961-66. (doi: 10.1126/science.1086478).

23. Kucharski AJ, Camacho A, Checchi F, Waldman R, et al. Evaluation of the benefits and risks of introducing Ebola community care centers, Sierra Leone. Emerg Infect Dis., 2015;21(3):393-99. (doi: 10.3201/eid2103.141892).

24. Cooper BS, Pitman RJ, Edmunds WJ, Gay NJ. Delaying the international spread of pandemic influenza. PLoS Med, 2006;3(6):e212. (doi: 10.1371/journal.pmed.0030212).

25. Anderson RM, May RM, Anderson B. Infectious Diseases of Humans: Dynamics and Control. oxford science publications, 1992.

26. Li Q, Guan X, Wu P, Wang X, et al. Early Transmission Dynamics in Wuhan, China, of Novel Coronavirus-Infected Pneumonia. NEJM, 2020;382:1199-207. (doi: 10.1056/NEJMoa2001316). 